\title{
Real gas effects in thermally choked nozzle flows
}

By Can F. Delale*, Max-Planck-Institut für Strömungsforschung, Bunsenstrasse $10 \cdot \mathrm{D}-3400$ Göttingen, Germany and Department of Mathematics, Bilkent University, 06533 Bilkent, Ankara, Turkey and Gerd E. A. Meier, DLR Institut für Experimentelle Strömungsmechanik, Bunsenstrasse 10 - D-3400 Göttingen, Germany

\section{Introduction}

When a condensible vapor, with or without a carrier gas, is expanded in a Laval nozzle, it crosses the saturation line without any noticeable phase change until sufficient number of condensation nuclei are created in the parent phase by homogeneous nucleation so that the delayed phase transition becomes visible (onset of condensation). When this happens, a considerable amount of heat of condensation is set free in the supersonic region giving rise to an increase in the pressure, temperature and density over a relatively small thickness. Figure 1 shows such an expansion in the pressuretemperature diagram of the vapor. The phenomenon described has been studied extensively in the literature (e.g. see Stever [1], Wegener and Mack [2], Wegener [3], Barschdorff [4], Gyarmathy [5] and their extensive references) and is sometimes inappropriately termed as 'condensation shock'. If the heat released by condensation exceeds a critical amount, the compressive effects from excessive heat release overweighs the influence of the increasing cross-section moving the flow Mach number ${ }^{1}$ toward unity. The flow then can no longer continue and is said to be thermally choked. In such a case an embedded normal shock wave occurs upstream due to compressive effects from enormous amount of heat addition. Early investigations dealing with thermal choking include those by Wegener and Mack [2], Pouring [6], Barschdorff [4] and Barschdorff and Fillipov [7]. All of these investigations emphasize the phenomenology rather than the mathematical theory. Actually it is only recently that the mathematical theory of thermal choking in nozzles has been discussed thoroughly [8] by exhibiting the necessary and sufficient conditions for a perfect condensible vapor.

* Alexander von Humboldt Fellow.

I The flow Mach number is usually taken as the local frozen Mach number. 
Figure 1

Typical expansion of the condensible vapor through nozzles in the pressure-temperature $\left(p_{v}^{\prime}-T^{\prime}\right)$ diagram ( $M$ is the flow Mach number, $T_{0}^{\prime}$ is the reservoir temperature, $T_{s}^{\prime}$ is the saturation temperature and $T_{k}^{\prime}$ is the onset temperature).

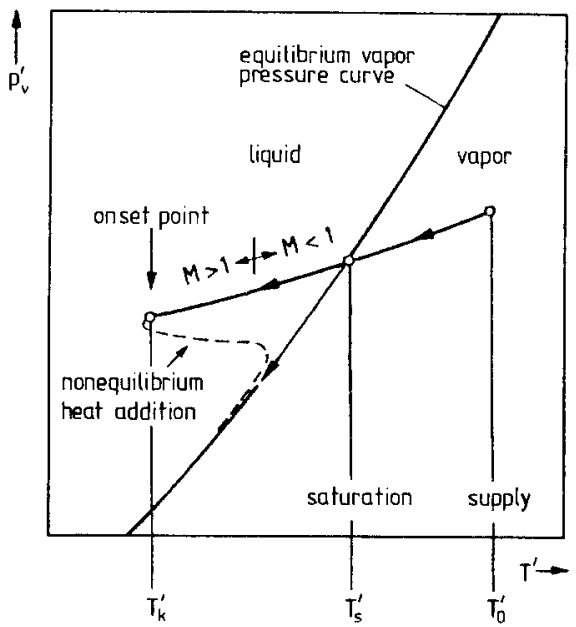

It is the aim of this paper to extend the theory to include real gas behavior of the condensible vapor. In spite of the fact that for most working fluids the inclusion of real gas effects turns out to be insignificant in the adiabatic expansion regions of nozzle flows with homogeneous condensation, it may influence the flow field in the heat addition zones significantly. Consequently the conditions for thermal choking stated for the case of a perfect condensible vapor may no longer be valid. Such situations are treated in this study in detail by accounting for real gas behavior of the condensible vapor from its virial equation of state truncated after the second virial coefficient. As a result thermal choking conditions which generalize those for a perfect condensible vapor are obtained.

\section{Flow and state equations}

We consider the transonic flow of a mixture of a carrier gas (denoted by subscript $i$ ) and a condensible vapor (denoted by subscript $v$ ) through a Laval nozzle with geometry as shown in Fig. 2 and with initial reservoir temperature $T_{0}^{\prime}$, initial specific humidity $\omega_{0}$ and initial relative humidity $\phi_{0}$.

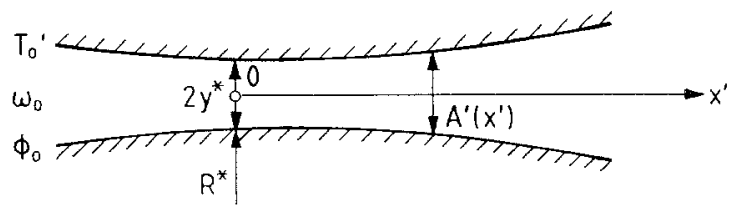

Figure 2

Geometry of a typical Laval nozzle. 
We adopt the homogeneous flow model with the conventional assumptions that there is no slip between the dispersed droplets and the mixture of gases and that the enthalpy difference between the liquid and vapor phases is approximated by the latent heat of condensation. We also neglect the heat transfer mechanism between the droplets and the surrounding gaseous phase. Furthermore the carrier gas is treated as a perfect gas with a thermal equation of state

$$
p_{i}^{\prime}=\frac{\Re}{\mu_{i}} \varrho_{i}^{\prime} T^{\prime}
$$

where $\mathfrak{R}$ is the universal gas constant, $T^{\prime}$ is the temperature of the mixture, $\mu_{i}$ is the molecular weight of the carrier gas, and $p_{i}^{\prime}$ and $\varrho_{i}^{\prime}$ are respectively the partial pressure and the density of the carrier gas. For the condensible vapor phase we assume a virial equation of state in the form

$$
\frac{p_{v}^{\prime}}{\varrho_{v}^{\prime}}=\frac{\Re}{\mu_{v}} T^{\prime}+\beta^{\prime}\left(T^{\prime}\right) p_{v}^{\prime}
$$

where $\mu_{v}$ is the molecular weight of the vapor, $p_{v}^{\prime}$ and $\varrho_{v}^{\prime}$ are respectively the partial vapor pressure and the vapor density, and $\beta^{\prime}\left(T^{\prime}\right)$ is the second virial coefficient ${ }^{2}$ (the second virial coefficients of gases can be found in [9]). If we denote the condensate mass fraction (the ratio of the mass flow rate of condensate to that of the mixture) by $g$, we then have

$$
\varrho_{v}^{\prime}=\varrho^{\prime}\left(\omega_{0}-g\right)
$$

and

$$
\varrho_{i}^{\prime}=\varrho^{\prime}\left(1-\omega_{0}\right)
$$

where $\varrho^{\prime}$ is the density of the mixture. Utilizing equations (1)-(4), we arrive by Dalton's law $\left(p^{\prime}=p_{v}^{\prime}+p_{i}^{\prime}\right)$ at the thermal equation of state of the mixture of a perfect carrier gas and a condensible vapor treated as a real gas in the form

$$
p^{\prime}=\frac{\Re}{\mu_{0}} \varrho^{\prime} T^{\prime}\left[\frac{1-g \mu_{0} / \mu_{v}-\left(1-\omega_{0} \mu_{0} / \mu_{v}\right)\left(\omega_{0}-g\right) \beta^{\prime} \varrho^{\prime}}{1-\left(\omega_{0}-g\right) \beta^{\prime} \varrho^{\prime}}\right]
$$

where $\mu_{0}$, the molecular weight of the mixture in the reservoir, is defined by

$$
\frac{1}{\mu_{0}} \equiv \frac{1-\omega_{0}}{\mu_{i}}+\frac{\omega_{0}}{\mu_{v}}
$$

To be able to discuss real gas effects on thermal choking in nozzle flows, the thermal equation of state (5) of the mixture should be accompanied by

2 Some authors assume a virial expansion in density instead of a virial expansion in pressure employed herein. The corresponding second virial coefficients are then different, but can easily be related. 
the inviscid equations of nozzle flows, namely

$$
\begin{aligned}
& \varrho^{\prime} u^{\prime} A^{\prime}=\text { constant } \\
& \left(p^{\prime}+\varrho^{\prime} u^{\prime 2}\right) A^{\prime}-\int p^{\prime} d A^{\prime}=\mathrm{constant}
\end{aligned}
$$

and

$$
h^{\prime}+\frac{1}{2} u^{\prime 2}=\text { constant }
$$

where $h^{\prime}$ is the specific enthalpy of the mixture (for its evaluation see the appendix), $u^{\prime}$ is the flow velocity and $A^{\prime}$ is the cross-sectional area of the nozzle. As is well known for nozzle flows with heat addition from condensation, the state of the condensible vapor crosses the saturation line without any noticeable phase change and the mixture expands almost isentropically in a metastable state unless a significant number of condensation nuclei are formed by homogeneous nucleation so that the phase change in the vapor phase becomes visible (onset of condensation) and a considerable amount of latent heat begins to be added to the flow (see Fig. 1). Since in this investigation we are primarily interested in heat addition to the flow from phase change, we choose our reference state as the saturation state of the vapor (which we denote by subscript $s$ ) and carry out the normalization

$$
p \equiv \frac{p^{\prime}}{p_{s}^{\prime}}, \quad T \equiv \frac{T^{\prime}}{T_{s}^{\prime}}, \quad \varrho \equiv \frac{\varrho^{\prime}}{\varrho_{s}^{\prime}}, \quad \beta \equiv \frac{\beta^{\prime}}{\beta_{s}^{\prime}},
$$

together with

$$
u \equiv \frac{u^{\prime}}{\sqrt{\Re T_{s}^{\prime} / \mu_{0}}} \quad \text { and } A=\frac{A^{\prime}}{A_{s}^{\prime}} .
$$

We also normalize the axial nozzle coordinate $x^{\prime}$ with respect to the throat height $2 y^{*}$ (see Fig. 2) as

$$
x \equiv \frac{x^{\prime}}{2 y^{*}} .
$$

The flow equations (7) -(9) together with the thermal equation of state (5) then take the normalized form

$$
\begin{aligned}
& \varrho u A=u_{s} \\
& p A+\lambda_{s} u_{s} u=1+\lambda_{s} u_{s}^{2}+R(g, x) \\
& \left\{\frac{1-\left[1-(1+\zeta) \omega_{0} \mu_{0} /\left(\mu_{v} c_{p 0}\right)\right]\left(\omega_{0}-g\right) \kappa_{s} \beta \varrho}{1-\left(\omega_{0}-g\right) \kappa_{s} \beta \varrho}\right\} T-\frac{q(g, x)}{c_{p 0}}+\frac{u^{2}}{2 c_{p 0}}=T_{r}
\end{aligned}
$$




$$
p=\lambda_{s} \varrho T\left\{\frac{1-g \mu_{0} / \mu_{v}-\left(1-\omega_{0} \mu_{0} / \mu_{v}\right)\left(\omega_{0}-g\right) \kappa_{s} \beta \varrho}{1-\left(\omega_{0}-g\right) \kappa_{s} \beta \varrho}\right\}
$$

with

$$
\begin{aligned}
& \kappa_{s} \equiv \omega_{s}^{\prime} \varrho_{s}^{\prime} \leq 0, \\
& \lambda_{s} \equiv \frac{1-\omega_{0} \kappa_{s}}{1-\left(1-\omega_{0} \mu_{0} / \mu_{v}\right) \omega_{0} \kappa_{s}}, \\
& \zeta \equiv-\beta^{-1} T \frac{d \beta}{d T} \geq 0
\end{aligned}
$$

and where

$$
\begin{aligned}
& R(g, x) \equiv \int_{x_{s}}^{x} p \frac{d A}{d \xi} d \xi \\
& \frac{q(g, x)}{c_{p 0}} \equiv H \frac{g L}{c_{p 0}}, \\
& T_{r} \equiv T_{0}+\frac{\omega_{0} \beta_{0}^{\prime}\left(1+\zeta_{0}\right) \phi_{0} p_{\text {sat }}^{\prime}\left(T_{0}^{\prime}\right)}{c_{p 0}^{\prime} T_{s}^{\prime}}
\end{aligned}
$$

with $L \equiv L^{\prime} / L_{s}^{\prime}$ where $L^{\prime}$ denotes the latent heat of condensation, $T_{0} \equiv$ $T_{0}^{\prime} / T_{s}^{\prime}, H \equiv \mu_{v} L_{s}^{\prime} /\left(\mathfrak{R} T_{s}^{\prime}\right), p_{\text {sat }}^{\prime}\left(T_{0}^{\prime}\right)$ is the saturation pressure of the vapor in the reservoir, $\beta_{0}^{\prime}$ is the second virial coefficient of the vapor in the reservoir, $\zeta_{0}$ is the value of $\zeta$ at the reservoir, $c_{p 0}^{\prime}$ is the specific heat of the mixture (for its definition see the appendix) and $c_{p 0} \equiv c_{p 0}^{\prime} \mu_{0} / \mathfrak{R}$.

It is obvious that the system of equations (13)-(16) does not form a complete system unless it is supplemented by the nonequilibrium integral condensation rate equation $[10,11]$. However, as has been discussed in great detail by Delale et al. [8], for the analysis of thermal choking it suffices to consider the function $g=g(x)$ as an arbitrary, positive, strictly increasing function of $x$ for $x>x_{s}$. With this assertion equations (13)-(16) form an integro-algebraic system for the variables $u, p, T$ and $\varrho$ depending on the condensate mass fraction $g=g(x)$ and the normalized area $A=A(x)$. Manipulations then yield a cubic equation for the functional $u=u(g, x)$ as

$$
u^{3}+B_{2} u^{2}+B_{1} u+B_{0}=0
$$

where

$$
\begin{aligned}
B_{2} \equiv & -\frac{2 \gamma\left[1+\lambda_{s} u_{s}^{2}+R(g, x)\right]}{\lambda_{s} u_{s}\left[\gamma+1+(\gamma-1) g \mu_{0} / \mu_{v}\right]} \\
& -\frac{u_{s}\left(\omega_{0}-g\right) \kappa_{s} \beta}{A} \frac{\left[\gamma+1-(\gamma-1)(1+2 \zeta) \omega_{0} \mu_{0} / \mu_{v}\right]}{\left[\gamma+1+(\gamma-1) g \mu_{0} / \mu_{v}\right]}
\end{aligned}
$$




$$
\begin{aligned}
B_{1} \equiv & \frac{2 \gamma\left(1-g \mu_{0} / \mu_{v}\right)}{\left[\gamma+1+(\gamma-1) g \mu_{0} / \mu_{v}\right]}\left[T_{r}+\frac{q(g, x)}{c_{p 0}}\right] \\
& +\frac{2 \kappa_{s} \beta}{\lambda_{s} A} \frac{\left[\gamma-(\gamma-1)(1+\zeta) \omega_{0} \mu_{0} / \mu_{v}\right]\left(\omega_{0}-g\right)}{\left[\gamma+1+(\gamma-1) g \mu_{0} / \mu_{v}\right]}\left[1+\lambda_{s} u_{s}^{2}+R(g, x)\right]
\end{aligned}
$$

and

$$
B_{0} \equiv-\frac{2 \gamma u_{s}\left(1-\omega_{0} \mu_{0} / \mu_{v}\right)\left(\omega_{0}-g\right) \kappa_{s} \beta}{\left[\gamma+1+(\gamma-1) g \mu_{0} / \mu_{v}\right] A}\left[T_{r}+\frac{q(g, x)}{c_{p 0}}\right]
$$

where the adiabatic exponent $\gamma$ of the mixture is defined by $\gamma \equiv c_{p 0} /\left(c_{p 0}-1\right)$. The solution of the cubic equation (23) relates the function $u=u(g, x)$ of the mixture to the functional $R(g, x)$ and to the functions $\beta=\beta(T)$ and $L=L(T)$. The functional relations for the rest of the flow variables then follow in a natural way:

$$
\begin{aligned}
\varrho(g, x)= & \frac{u_{s}}{A(x) u(g, x)}, \\
T(g, x)= & {\left[T_{r}+\frac{q(g, x)}{c_{p 0}}-\frac{u^{2}(g, x)}{2 c_{p 0}}\right] } \\
& \times\left[\frac{1-\left(\omega_{0}-g\right) \kappa_{s} \beta \varrho(g, x)}{1-\left[1-(1+\zeta) \mu_{0} \omega_{0} /\left(\mu_{v} c_{p 0}\right)\right]\left(\omega_{0}-g\right) \kappa_{s} \beta \varrho(g, x)}\right]
\end{aligned}
$$

and

$$
\begin{aligned}
p(g, x)= & \lambda_{s} \varrho(g, x) T(g, x) \\
& \times\left[\frac{1-g \mu_{0} / \mu_{v}-\left(1-\omega_{0} \mu_{0} / \mu_{v}\right)\left(\omega_{0}-g\right) \kappa_{s} \beta \varrho(g, x)}{1-\left(\omega_{0}-g\right) \kappa_{s} \beta \varrho(g, x)}\right] .
\end{aligned}
$$

Equations (23) - (29) simplify considerably for the nozzle flow of a pure condensible vapor $\left(\omega_{0}=1\right)$. In such a case by equation (26) we have $B_{0}=0$ and the cubic equation (23), aside from the trivial solution $u=0$, yields the functional relation

$$
\begin{aligned}
u(g, x)= & \frac{2 \gamma}{[\gamma+1+(\gamma-1) g]}\left\{\frac{\left[1+\lambda_{s} u_{s}^{2}+R(g, x)\right]}{2 \lambda_{s} u_{s}}\right. \\
& \left.+\frac{u_{s}[1-(\gamma-1) \zeta](1-g) \kappa_{s} \beta}{2 \gamma A} \pm \sqrt{\Delta(g, x)}\right\}
\end{aligned}
$$

where $\lambda_{s}$ simplifies as $\lambda_{s}=1-\kappa_{s}, \gamma$ is now the adiabatic exponent of the vapor and the functional $\Delta(g, x)$ is defined by

$$
\Delta(g, x) \equiv T_{r} \Theta(g)\left[\frac{q^{*}(g, x)}{c_{p v} T_{r}}-\frac{q(g, x)}{c_{p v} T_{r}}\right]
$$


with the critical amount of heat $q^{*}(g, x)$ not to be exceeded for a continuous solution and the function $\Theta(g, x)$ defined by

$$
\begin{aligned}
& \frac{q^{*}(g, x)}{c_{p v} T_{r}} \\
& \equiv \frac{\left\{\left[1+\lambda_{s} u_{s}^{2}+R(g, x)\right] /\left(2 \lambda_{s} u_{s}\right)+u_{s}[1-(\gamma-1) \zeta](1-g) \kappa_{s} \beta /(2 \gamma A)\right\}^{2}}{T_{r} \Theta(g)} \\
& \quad-1-\frac{[1-(\gamma-1) \zeta]\left[1+\lambda_{s} u_{s}^{2}+R(g, x)\right] \kappa_{s} \beta}{\gamma \lambda_{s} A T_{r}} \\
& \Theta(g) \equiv \frac{[\gamma+1+(\gamma-1) g](1-g)}{2 \gamma}
\end{aligned}
$$

and with $c_{p v} \equiv \gamma /(\gamma-1)$ denoting the normalized specific heat of the vapor and $T_{r}$ given by equation (22) in the limit as $\omega_{0} \rightarrow 1$. The functional relations (27-(29) for this case can easily be obtained by setting $\omega_{0}=1$ (consequently $\mu_{0}=\mu_{v}$ and $c_{p 0}=c_{p v}$ ).

It should be noticed that a solution of the cubic equation (23) for $u$ in the case of a condensible vapor and carrier gas and of the functional relation (30) together with equations (31)-(33) for $u$ in the case of a pure condensible vapor and the functional relations $(27)-(29)$ do not yield an implicit algebraic solution of the flow field even if the function $g=g(x)$ is supplied by some means (e.g. empirically) since the functional $R(g, x)$ remains to be evaluated. The complete solution needs a detailed treatment of the condensation rate equation coupled to the equations of flow and state, and we will not discuss it any further. Instead we will emphasize how real gas effects from the second virial coefficient of the condensible vapor alter the conditions for thermal choking previously derived in [8] for the case of a mixture of a perfect carrier gas and a perfect condensible vapor.

\section{Thermal choking of real gas flows in nozzles}

For nozzle flows with nonequilibrium condensation, the effect of heat addition to the flow becomes important downstream of the onset of condensation, i.e. for $x>x_{k}$ where $x_{k}$ denotes the onset of condensation (this point can be located either empirically [12], semi-empirically by similarity analysis [13], numerically [14] or by the asymptotic solution of the condensation rate equation $[11,15])$. We herein assume that heat addition to the flow from condensation occurs in the supersonic region of the nozzle so that $x_{k}>0$. As long as the amount of heat added to the flow does not exceed a certain value, called the critical amount, the flow field remains continuous (subcritical flow). Once the critical amount is exceeded, the flow is said to 
be thermally choked and no continuous solution of the flow is possible anymore. In this case the inclusion of an embedded frozen gasdynamic normal shock wave arising from compressive effects due to excessive heat addition becomes necessary (supercritical flow). The necessary and sufficient conditions for thermal choking in nozzle flows where the condensible vapor is treated as a perfect gas are recently exhibited in Delale et al. [8]. In this section we generalize these conditions to account for the influence of the real gas behavior of the condensible vapor on the phenomenon of thermal choking. Although real gas effects are usually thought to be weak enough to be neglected in adiabatic nozzle flows, they may influence the flow field in the heat addition region for some working fluids and consequently may alter the thermal choking conditions previously derived [8]. In what follows we study these effects utilizing the virial equation of state of the condensible vapor truncated after the second virial coefficient.

We first discuss the general case of a mixture of a condensible vapor (treated now as a real gas) and a perfect carrier gas. In particular we concentrate on the solution of the cubic equation (23) of the preceding section for the real functional $u=u(g, x)$. From the fundamental theorem of algebra, equation (23) has precisely three roots (denoted by $u_{1}, u_{2}$ and $u_{3}$ ) over the complex field and at least one of the roots is real. Since $B_{0} \geq 0$ by equation (26), we have $u_{1} u_{2} u_{3}=-B_{0} \leq 0$ which implies that at least one of the roots, say $u_{1}$, is negative definite (i.e. $u_{1} \leq 0$ ). This solution $u_{1}(g, x)$, however, does not correspond to any physical solution of the flow field and therefore must be discarded. It is well-known that the other two roots $\left(u_{2}\right.$ and $\left.u_{3}\right)$ of equation (23) are real and distinct if $s^{3}+t^{2}<0$, real and repeated if $s^{3}+t^{2}=0$ and complex conjugate if $s^{3}+t^{2}>0$ where

$$
s \equiv \frac{1}{3} B_{1}-\frac{1}{9} B_{2}^{2}
$$

and

$$
t \equiv \frac{1}{6}\left(B_{1} B_{2}-3 B_{0}\right)-\frac{1}{27} B_{2}^{3} .
$$

From the relations $u_{1}+u_{2}+u_{3}=-B_{2}>0$ and $u_{1} \leq 0$, it can further be shown that both $u_{2}$ and $u_{3}$ are positive if they are real. We recall that $B_{0}, B_{1}$ and $B_{2}$ given by equations (24) $-(26)$ are all real continuous functionals depending on $g=g(x)$ and $A=A(x)$; therefore, if a real solution $u=u(g, x)$ of equation (23) exists, then it should also be continuous. It follows from the last two statements that the condition $s^{3}+t^{2} \leq 0$ corresponds to the case of a continuous positive solution $u=u_{2}$ or $u=u_{3}$ (subcritical flow). In particular the smaller of the roots, say $u_{2}\left(u_{2} \leq u_{3}\right)$, yields the flow velocity 
in the 'subsonic' region and the greater one $\left(u_{3}\right)$ in the 'supersonic' region'. The condition $s^{3}+t^{2}=0$ or equivalently $u_{2}=u_{3}$ may be reached at one or more points along the nozzle axis and corresponds to a critical condition ${ }^{4}$ where the flow bifurcates into two branches: 'subsonic' or 'supersonic'. On the other hand if $s^{3}+t^{2}>0$ for some $x>x_{k}$, then $u_{2}$ and $u_{3}$ are complex conjugate at that location and no continuous physical solution exists. The flow in this case is termed thermally choked. When this happens, there must be a point $\hat{x}>x_{k}$ upstream where the flow reaches the critical condition $s^{3}+t^{2}=0$. Thus we have proved the following result:

Proposition 3.1. For the expansion of a mixture of a condensible real vapor and a perfect carrier gas in nozzles, a necessary condition for thermal choking is that the critical flow condition $s^{3}+t^{2}=0$ is reached at some point $\hat{x}>x_{k}$ along the nozzle axis.

To discuss sufficiency we now assume that the flow becomes critical at some point $\hat{x}>x_{k}$. Then there are two possibilities downstream of the point $\hat{x}$ : either $s^{3}+t^{2}>0$ or $s^{3}+t^{2}<0$. If $s^{3}+t^{2}<0$ downstream of the point $\hat{x}$, the flow then is subcritical. Conversely if $s^{3}+t^{2}>0$ downstream of the point $\hat{x}$, the flow can not continue and is thermally choked. Since the condition

$$
\frac{d}{d x}\left(s^{3}+t^{2}\right)>0
$$

at $x=\hat{x}$ would imply the condition $s^{3}+t^{2}>0$ downstream at the point $\hat{x}$, we have the following result:

Proposition 3.2. A sufficient condition for the expansion of a mixture of a condensible real vapor and a perfect carrier gas through nozzles to be thermally choked is that

$$
s^{3}+t^{2}=0 \text { and } \frac{d}{d x}\left(s^{3}+t^{2}\right)>0
$$

at some point $\hat{x}>x_{k}$ along the nozzle axis.

The conditions stated in Propositions 3.1 and 3.2 become more informative and simple for the case of a pure condensible vapor $\left(\omega_{0}=1\right)$. In this

\footnotetext{
3 The use of the words 'subsonic' and 'supersonic' needs some caution since there is no unique definition of the speed of sound in the two-phase dispersed droplet regime of the condensation zones.

4 This condition may as well be called a 'sonic' condition.
} 
limit we have

$$
s^{3}+t^{2}=-\frac{4}{27} C^{2}\left[\frac{\gamma}{\gamma+1+(\gamma-1) g}\right] \Delta(g, x)
$$

where $\Delta(g, x)$ is given by equation (31) and

$$
\begin{aligned}
C \equiv & \frac{2 \gamma(1-g)}{[\gamma+1+(\gamma-1) g]} \\
& \times\left\{T_{r}+\frac{q(g, x)}{c_{p v}}+\frac{[1-(\gamma-1) \zeta] \kappa_{s} \beta}{\gamma\left(1-\kappa_{s}\right) A}\left[1+\left(1-\kappa_{s}\right) u_{s}^{2}+R(g, x)\right]\right\} .
\end{aligned}
$$

By equation (36) Propositions 3.1 and 3.2 now simplify as

Proposition 3.3. A necessary condition for the expansion in nozzles of a condensible pure vapor treated as a real gas to be thermally choked is that

$$
\Delta=0
$$

or equivalently

$$
\frac{q^{*}}{c_{p v} T_{r}}=\frac{q}{c_{p v} T_{r}}
$$

at some point $x=\hat{x}$ along the nozzle axis where $q(g, x)$ is the amount of latent heat added to the flow given by equation (21) and $q^{*}(g, x)$ is the critical amount of heat given by equation (32).

Proposition 3.4. A sufficient condition for the expansion in nozzles of a condensible pure vapor treated as a real gas to be thermally choked is that

$$
\Delta=0 \text { and } \frac{d \Delta}{d x}<0
$$

or equivalently

$$
\frac{q^{*}}{c_{p v} T_{r}}=\frac{q}{c_{p v} T_{r}} \quad \text { and } \quad \frac{d}{d x}\left(\frac{q^{*}}{c_{p v} T_{r}}\right)<\frac{d}{d x}\left(\frac{q}{c_{p v} T_{r}}\right)
$$

at some point $x=\hat{x}$ along the nozzle axis.

We should finally mention that in the limit when the condensible vapor is treated as a perfect gas $\left(\beta^{\prime} \rightarrow 0\right)$, it is straightforward to show that the results stated in Propositions 3.1-3.4 reduce precisely to the conditions stated in Delale et al. [8]. 


\section{Concluding remarks}

The thermal choking conditions in nonequilibrium condensing nozzle flows with or without a perfect carrier gas are investigated by taking into account real gas behavior of the condensible vapor by the second virial coefficient. The conditions obtained recently for thermal choking of nozzle flows of a perfect condensible vapor, with or without a carrier gas, [8] are therefore generalized. In the case of a mixture of a condensible real vapor and a perfect carrier gas, the critical amount of heat necessary to thermally choke the flow can only be defined implicitly by the condition given in Proposition 3.1. However, for the case of a pure condensible vapor treated as a real gas the critical amount of heat to thermally choke the flow is explicitly defined by equation (32).

It can clearly be seen that real gas effects of the condensible vapor can influence the flow field especially in the condensation zones where heat addition is significant. Although for condensing nozzle flows it seems sufficient to take into account real gas behavior by the second virial coefficient of the condensible vapor, it may be interesting to compare the conditions achieved herein with those to be obtained by different equations of state (e.g. the van der Waals equation of state). However, the system of equations obtained by these different equations of state generally turns out to be too complicated to yield any information on the conditions of thermal choking in nozzles.

\section{Appendix: The specific enthalpy of a mixture of a condensible vapor and a carrier gas}

The specific enthalpy of the vapor phase $h_{v}^{\prime}$ can be evaluated from

$$
d h_{v}^{\prime}=\left(c_{v}^{\prime}\right)_{v} d T^{\prime}+\frac{d p_{v}^{\prime}}{\varrho_{v}^{\prime}}-T^{\prime}\left(\frac{\partial p_{v}^{\prime}}{\partial T^{\prime}}\right)_{\varrho_{v}^{\prime}} \frac{d \varrho_{v}^{\prime}}{\varrho_{v}^{\prime 2}}
$$

where $\left(c_{v}^{\prime}\right)_{v}$ is the specific heat of the vapor at constant volume. Utilizing the thermal equation of state (2) for the vapor we arrive at

$$
d h_{v}^{\prime}=c_{p v}^{\prime} d T^{\prime}+\left[\beta^{\prime}-T^{\prime} \frac{d \beta^{\prime}}{d T^{\prime}}\right] d p_{v}^{\prime}
$$

where $c_{p v}^{\prime}$ is the specific heat of the vapor at constant pressure given by

$$
c_{p v}^{\prime}=\left(c_{v}^{\prime}\right)_{v}+\frac{\Re}{\mu_{v}}\left[\frac{1-\left(\beta^{\prime}-T^{\prime} \frac{d \beta^{\prime}}{d T^{\prime}}\right) \varrho_{v}^{\prime}}{1-\beta^{\prime} \varrho_{v}^{\prime}}\right]^{2} .
$$

It can be shown that $\left(c_{v}^{\prime}\right)_{v}$ is only a function of temperature for a vapor 
obeying the thermal equation of state given by equation (2). Even so, equation (A2) is not readily integrable since by equation (A3) we have $c_{p v}^{\prime}=c_{p v}^{\prime}\left(T^{\prime}, \varrho_{v}^{\prime}\right)$. However, simplification of equation (A3) is possible if we consider the nucleating flow states. Although for these states both $\beta^{\prime}$ and $T^{\prime} d \beta^{\prime} / d T^{\prime}$ are of the same order of magnitudes for most fluids, both $\left|\beta^{\prime}\right| \varrho_{v}^{\prime}$ and $\left|T^{\prime} d \beta^{\prime}\right| d T^{\prime} \mid \varrho_{v}^{\prime}$ are small enough to be neglected compared to unity in equation (A3). This suggests that for most fluids in nucleating flow we can take

$$
c_{p v}^{\prime} \approx c_{p v}^{\prime}\left(T^{\prime}\right)=\left(c_{v}^{\prime}\right)_{v}+\frac{\Re}{\mu_{v}} .
$$

On the other hand, in spite of the fact that $\beta^{\prime}$ and $T^{\prime} d \beta^{\prime} / d T^{\prime}$ show strong dependence on temperature in the nucleating flow states, the functions $\beta^{\prime}-T^{\prime} d \beta^{\prime} / d T^{\prime}$ and $c_{p v}^{\prime}\left(T^{\prime}\right)$, nevertheless, do not strongly depend on temperature and can be approximated by their mean values over the operational temperature range of the nozzle ${ }^{5}$. With these approximations in mind, equation (2) can now be integrated to yield

$$
h_{v}^{\prime}=c_{p v}^{\prime} T^{\prime}+\left[\beta^{\prime}-T^{\prime} \frac{d \beta^{\prime}}{d T^{\prime}}\right] p_{v}^{\prime}+\text { constant. }
$$

On the other hand, for the specific enthalpy $h_{i}^{\prime}$ of the perfect carrier gas we have

$$
h_{i}^{\prime}=c_{p i}^{\prime} T^{\prime}+\text { constant }
$$

where $c_{p i}^{\prime}$ is the specific heat of the perfect carrier gas at constant pressure. The specific enthalpy of the mixture is then obtained by

$$
h^{\prime}=\omega_{0} h_{v}^{\prime}+\left(1-\omega_{0}\right) h_{i}^{\prime}-g L^{\prime}
$$

which evaluates to

$$
h^{\prime}=c_{p 0}^{\prime} T^{\prime}+\omega_{0}\left(\beta^{\prime}-T^{\prime} \frac{d \beta^{\prime}}{d T^{\prime}}\right) p_{v}^{\prime}-g L^{\prime}+\text { constant }
$$

where $c_{p 0}^{\prime}$ is the specific heat of the mixture defined by

$$
c_{p 0}^{\prime} \equiv \omega_{0} c_{p v}^{\prime}+\left(1-\omega_{0}\right) c_{p i}^{\prime}
$$

and $L^{\prime}$ is the latent heat of condensation. Utilizing equations (2) and (3), we finally arrive at

$$
h^{\prime}=\left[c_{p 0}^{\prime}+\frac{\Re \omega_{0}}{\mu_{v}} \frac{\left(\omega_{0}-g\right)\left(\beta^{\prime}-T^{\prime} \frac{d \beta^{\prime}}{d T^{\prime}}\right) \varrho^{\prime}}{1-\left(\omega_{0}-g\right) \beta^{\prime} \varrho^{\prime}}\right] T^{\prime}-g L^{\prime}+\text { constant } .
$$

5 Treating $\beta^{\prime}-T^{\prime} d \beta^{\prime} / d T^{\prime}$ as a constant is also possible. This then corresponds to $\beta^{\prime}$ linearly varying with temperature. 


\title{
Acknowledgements
}

The authors are grateful to Dr. A. Dillmann for valuable discussions. One of us (C.F.D.) would like to thank Professor E. A. Müller for his hospitality and the Alexander von Humboldt Foundation for their generous support during his stay at MPI für Strömungsforschung in Göttingen.

\section{References}

[1] H. G. Stever, in Fundamentals of Gas Dynamics. High Speed Aerodynamics and Jet Propulsion, vol. III. Princeton University Press 1958.

[2] P. P. Wegener and L. M. Mack, in Adv. Appl. Mech., vol. V. Academic Press, New York 1958.

[3] P. P. Wegener, in Nonequilibrium Flows, ed. P. P. Wegener. Marcel Dekker, New York 1969.

[4] P. G. Hill, J. Fluid Mech. 76, 593 (1966).

[5] G. Gyarmathy, Rev. Fr. Mec. 57, 35 (1976).

[6] A. A. Pouring, Phys. Fluids 8, 1802 (1965).

[7] D. Barschdorff and G. A. Fillipov, Heat Transfer-Soviet Research 2(5), 76 (1970).

[8] C. F. Delale, G. H. Schnerr and J. Zierep, The mathematical theory of thermal choking in nozzle flows. ZAMP 44, 943-976 (1993).

[9] J. H. Dymond and E. B. Smith, The Virial Coefficients of Pure Gases and Liquids. Clarendon, Oxford 1980.

[10] K. Oswatitsch, Z. angew, Math. Mech. 22, 1 (1942).

[11] J. H. Clarke and C. F. Delale, Phys. Fluids 29, 1398 (1986).

[12] G. H. Schnerr, Expts. in Fluids 7, 145 (1989).

[13] J. Zierep and S. Lin, Forsch. Ing. Wes. 33, 169 (1967).

[14] G. H. Schnerr and U. Dohrmann, in Proc. IUTAM Symposium Transsonicum III, ed. J. Zierep and H. Oertel. Springer, Berlin 1989.

[15] C. F. Delale, G. H. Schnerr and J. Zierep, Asymptotic Solution of Transonic Nozzle Flows with Homogeneous Condensation. I. Subcritical Flows, to appear in Phys. Fluids A (1993).

\begin{abstract}
Real gas effects in condensing nozzle flows are discussed by the virial equation of state truncated after the second virial coefficient. The thermal choking conditions in nozzles previously derived for a perfect condensible vapor are generalized to include real gas effects. For these cases it is shown that the critical amount of heat necessary to thermally choke the flow can be defined explicitly only for the expansion of a pure vapor.
\end{abstract}

(Received: February 22, 1993) 